\title{
Stability of pro- and anti-inflammatory immune biomarkers for human cohort studies
}

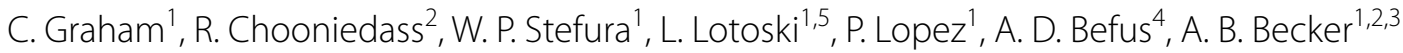 \\ and K. T. HayGlass 1,2,3* $^{*}$
}

\begin{abstract}
Background: Although discovery research has identified the importance of dozens of pro- and anti-inflammatory immune mediators in the pathogenesis, maintenance, exacerbation and resolution of inflammatory diseases, most human cohort studies have incorporated few or no immunological intermediate phenotypes in their analyses. Significant hindrances have been (1) the limited panel of biomarkers known to be readily detected in healthy human populations and (2) the stability, hence utility, of such biomarkers to repeated analysis.

Methods: The frequency and stability of 14 plasma biomarkers linked to in vivo immune regulation of allergic and autoimmune inflammatory disorders was determined in 140 healthy pediatric and adult participants. The impact of initial and multiple subsequent freeze/thaw cycles on pro-inflammatory (CCL2, CXCL10, IL-18, TNFa, IL-6), anti-inflammatory (IL-10, sTNF-RII, IL-1Ra), acute phase proteins (CRP, PTX3) and other biomarkers (sST2, IL-1RAcP) was subsequently quantified.

Results: Multiple biomarkers capable of providing an innate immune signature of inflammation were readily detected directly ex vivo in healthy individuals. These biomarker levels were unaffected when comparing paired data sets from freshly obtained, never frozen plasma or serum and matched aliquots despite extensive freeze/thaw cycles. Neither age nor sex affected stability. Similarly, no quantitative differences were found following repetitive analysis of inflammatory biomarkers in culture samples obtained following in vitro stimulation with TLR and RLR ligands.

Conclusions: A broad panel of in vivo and ex vivo cytokine, chemokine and acute phase protein biomarkers that have been linked to human chronic inflammatory disorders are readily detected in vivo and remain stable for analysis despite multiple freeze thaw cycles. These data provide the foundation and confidence for large scale analyses of panels of inflammatory biomarkers to provide better understanding of immunological mechanisms underlying health versus disease.
\end{abstract}

Keywords: Cohort, Inflammation, Human immunology, Immune regulation, Innate, Allergy, Autoimmunity

\section{Background}

Cohorts that range from hundreds to tens of thousands of individuals offer a powerful tool to validate, integrate and extend multidisciplinary findings obtained in basic biomedical discovery research. Currently, many human cohorts focus on identifying correlates of environmental variables (i.e. endotoxin exposure, birth order) with clinical outcomes. With few exceptions [1-3], only a small

\footnotetext{
*Correspondence: kent.hayglass@umanitoba.ca

${ }^{1}$ Department of Immunology, University of Manitoba, Winnipeg, MB, Canada

Full list of author information is available at the end of the article
}

proportion of such publications provide detailed examination of the intermediate phenotype of in vivo cytokine responses. For example, for allergic diseases, the most common chronic immune disorder in humans, PubMed identifies over 2000 publications for "birth cohort and allergy," but $<10 \%$ (155 citations) incorporate "cytokine or IL", and of these, only a subset examine inflammatory cytokines. Recent meta-analyses identify the knowledge gap resulting from this omission [4-8]. Given that most allergic and autoimmune disorders are driven by dysfunctional immune regulation, this identifies an important, but often missed, opportunity to enhance understanding 
of endotypes and mechanisms [9-12] underlying inflammatory diseases. The importance of doing so is increasingly clear [13-22].

A major logistical challenge that impedes more widespread adoption of such analyses is continuing uncertainty about the stability of immune biomarkers in plasma, serum or tissue culture samples to repeated analysis. Repetitive freezing and thawing $(\mathrm{F} / \mathrm{T})$ cycles can induce protein instability and aggregation [23, 24]. Among immune biomarkers in complex biological fluids (i.e. plasma, culture supernatants), some studies indicate extreme sensitivity, while others report that minimal variance results from the limited number of $\mathrm{F} / \mathrm{T}$ cycles that are typically required. This controversy may arise from the limited number of cytokine biomarkers that have been examined to date, the small sample sizes often utilized (i.e. frequently $<10$ individuals), the fact that several biomarkers examined are typically undetectable or at the limits of assay sensitivity in much of the healthy population examined (i.e. IL-6), hence substitution of spiked recombinant commercial proteins occurs as a surrogate for in vivo biomarkers. To an even greater extent than discovery based research projects which examine 20-40 individuals, cohorts face extensive logistical difficulties that prohibit analyses without repeated cycles of F/T [25-27].

Here we test the hypothesis that exposure of immune pro- and anti-inflammatory biomarkers to at least five repeated $\mathrm{F} / \mathrm{T}$ cycles introduces acceptable (i.e. minimal and predictable) variation. Using a panel of 14 proinflammatory (CCL2, CXCL10, IL-18, TNF $\alpha$, IL-6), anti-inflammatory (IL-10, sTNF-RII, IL-1Ra), acute phase proteins (CRP, PTX3) and other biomarkers (sST2, IL$1 \mathrm{RAcP}$ ) of inflammatory disorders previously linked to asthma, other atopic disorders [28-31] and a wide range of other chronic inflammatory conditions in humans, we quantify the sensitivity to initial freezing as well as to repeated F/T cycles that are inevitable if large studies incorporate analyses of immunological intermediate phenotypes. The findings demonstrate that a broad panel of pro-inflammatory, anti-inflammatory, acute phase proteins and other biomarkers of inflammatory diseases are readily amenable to analysis and should be more widely incorporated in large human cohort studies.

\section{Methods}

\section{Participants}

The Canadian Healthy Infant Longitudinal Development (CHILD) Study is a prospective longitudinal birth cohort of $>3500$ neonates [32]. In this report, following approval by the University of Manitoba Health Research Ethics Board, and written informed consent from each participant or their parent/guardian, non-fasting venous blood was obtained at the Winnipeg site to yield plasma and serum samples from 140 randomly selected participants (children and their parents).

\section{Sample preparation}

Peripheral blood was collected by venipuncture and used for plasma, serum and isolation of PBMC [33]. Briefly, samples were kept at room temperature during transport and prior to processing. Plasma was collected from 10-mL heparin Vacutainer tubes (BD, Mississauga, Canada) by centrifugation $(500 \mathrm{~g}, 10 \mathrm{~min})$. Serum was collected from 6-mL silica-coated Vacutainer tubes (BD) by centrifugation $(1000 g, 10 \mathrm{~min})$. Replicate plasma and serum sample aliquots $(300 \mu \mathrm{L}$ each) were prepared from each individual and then used within $24 \mathrm{~h}$ without freezing and were frozen at $-80{ }^{\circ} \mathrm{C}$ for one or five $\mathrm{F} / \mathrm{T}$ cycles before analysis. All analyses were performed comparing paired samples from the same individuals. Because the goal was to determine the impact of repeated changes of state (i.e. freeze/thawing) on plasma and supernatant samples, rather than how many months or years a sample could be stored and retain its integrity, all samples that underwent freeze thaw cycles were carried out within $24 \mathrm{~h}$ when comparing never frozen plasma or culture supernatant with samples subjected to one or five $\mathrm{F} / \mathrm{T}$ cycles. Paired analyses of one versus five $\mathrm{F} / \mathrm{T}$ cycles were carried out with storage at $-80{ }^{\circ} \mathrm{C}$ for a few days to a month in total. Samples were handled using standard laboratory conditions: thawed rapidly at $37{ }^{\circ} \mathrm{C}$ then kept on ice until analyzed.

\section{PBMC isolation and cell culture}

PBMC were prepared using Ficoll (GE Healthcare, Mississauga, Canada) and cultured (triplicates, 350,000 cells/ round bottom well in $200 \mu \mathrm{L}, 24 \mathrm{~h}$ ) in medium alone or with stimuli. Medium consisted of RPMI-1640 (Thermo Fisher Scientific, Mississauga, Canada) supplemented with $10 \%$ fetal bovine serum (GE Healthcare, Mississauga, Canada), 1\% L-glutamine (VWR International, Mississauga, Canada), 1\% Antibiotic-Antimycotic (Thermo Fisher Scientific), and 0.1\% 2-mercaptoethanol (Thermo Fisher Scientific). Innate stimuli used included TLR4 ligand LPS $(0.4 \mathrm{ng} / \mathrm{mL}$, InvivoGen, San Diego, CA) or RLR ligand Poly(I:C)/Lyovec $(250 \mathrm{ng} / \mathrm{mL}$, InvivoGen). All PBMC samples were cultured the day they were drawn. Supernatants were aliquoted and examined in parallel without ever freezing and after five $\mathrm{F} / \mathrm{T}$ cycles at -80 and $37^{\circ} \mathrm{C}$.

\section{Immunological assays}

All analyses were carried out in duplicate with paired samples after $0-5 \mathrm{~F} / \mathrm{T}$ cycles. $5 \%$ of sample pairs or triplets were repeated on a separate day. Meso Scale 
Discovery (MSD, Rockville, Maryland) singleplex assays were used to analyze plasma, serum and culture supernatants for CCL2, CRP, CXCL8, IL-6, IL-10, IL-18, IL$1 \mathrm{Ra}$, sTNF-RII and TNF $\alpha$ according to manufacturer's instructions. MSD V-Plex assays were used to analyze plasma and supernatant levels of CXCL10. ELISA (alkaline phosphatase-biotin coupled developing reagent with PNPP for development) was used to analyze plasma and serum levels of IL-1RAcP, PTX3, and sST2 (R\&D Systems, Minneapolis, Minnesota) using ultrasensitive protocols as previously described [34]. ELISAs incorporated four serial dilutions of each sample (i.e. 1/2, 1/4, 1/8 and $1 / 16$ ) that were assessed against eight serial dilutions of fresh aliquots of a constant recombinant lab standard stored at $-80{ }^{\circ} \mathrm{C}$ in individual $400 \mu \mathrm{L}$ aliquots (Cedarlane, Burlington, Canada; PeproTech, Quebec, Canada; $R \& D$ Systems). In most experiments, median coefficients of intra-assay variation between assays were below $5 \%$ for MSD assays and $10-15 \%$ for ELISA. Inter-assay variation was typically $<10-20 \%$.

\section{Statistics}

Data were analyzed using GraphPad Prism (La Jolla, California). Each point represents a single sample from an individual aliquot that has undergone the indicated number of F/T cycles. Mann-Whitney or Wilcoxon Matched Pairs/Signed Rank tests were used for unpaired and paired data sets respectively. While the multiple comparisons used in this study would normally require use of Bonferroni corrections, to obtain maximum sensitivity for detection of possible differences, significance was assessed at the lower threshold of a $95 \%$ confidence level (two-tailed $\mathrm{p}<0.05$ ).

\section{Results}

\section{Detection and stability of pro- and anti-inflammatory plasma biomarkers}

Freshly obtained plasma (or sera, see below) from a study population of 140 individuals of whom 30 were pediatric were examined. Adults were 20-44 years of age (median 32 year), while the pediatric population was aged 3 (median 3.2 year). Due to the number of biomarkers examined, and logistical constraints on how many independent assays could be performed simultaneously with never frozen samples, slightly different numbers of individuals were examined for each biomarker.

Figure 1a demonstrates that pro-inflammatory cytokines CCL2, CXCL10 and IL-18 are expressed at readily detectable levels in vivo in all of the individuals examined and can serve as readily detectable plasma

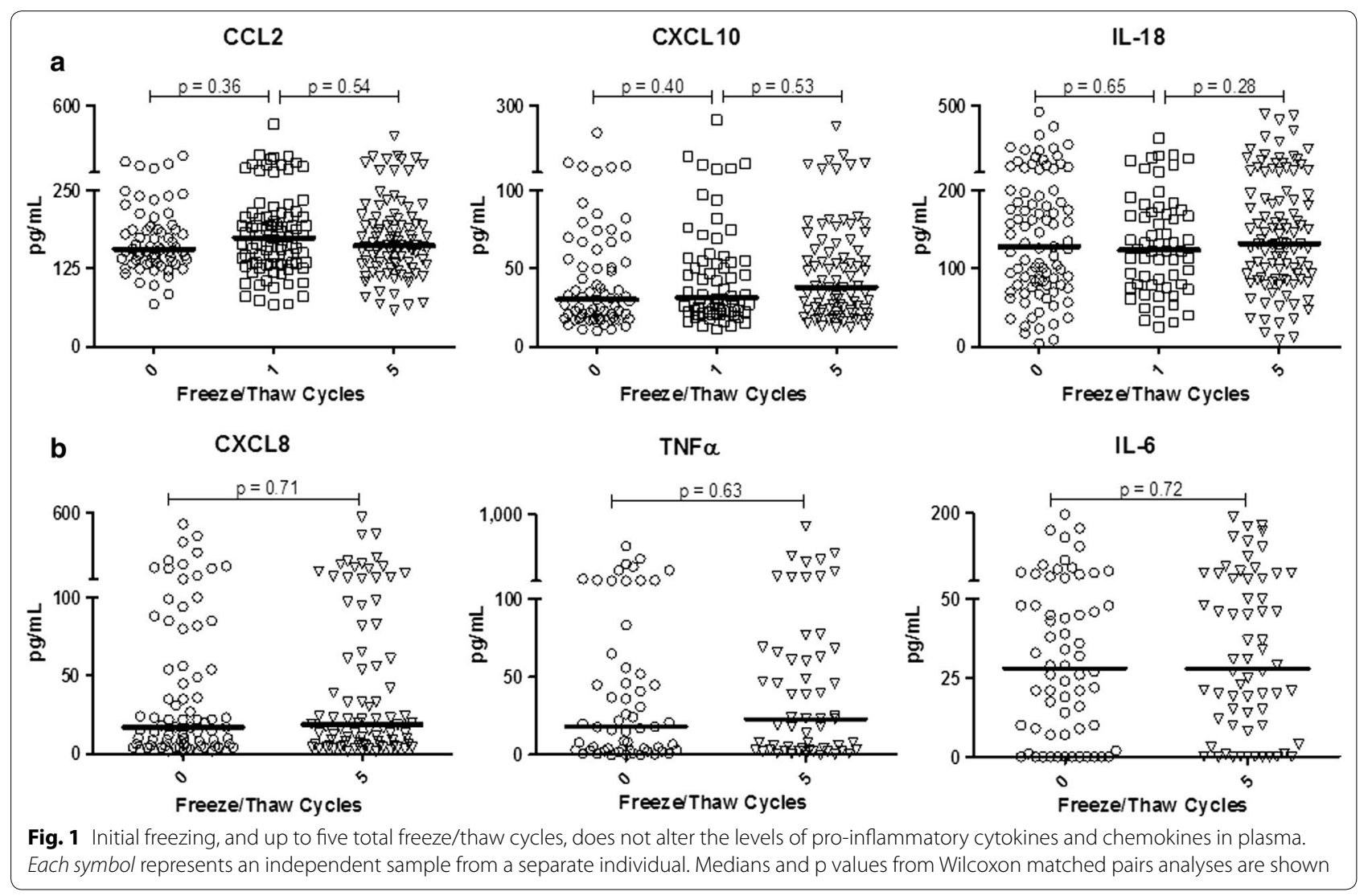


biomarkers in healthy human populations. When comparing fresh plasma with that from the same individuals after zero, one, or five F/T cycles (see "Methods" section), there was no detectable impact on the concentrations measured. Similarly, CXCL8, TNF $\alpha$ and IL- 6 values in never frozen plasma versus $5 \times \mathrm{F} / \mathrm{T}$ samples were indistinguishable $(\mathrm{p}>0.05)$ in paired longitudinal analyses (Fig. 1b).

Endogenous controls of inflammation, such as antiinflammatory cytokines and immune response modifiers, are pivotal yet are typically underrepresented in human studies of inflammation. Figure 2 reveals three points. Unlike pro-inflammatory biomarkers such as IL-6 and TNF $\alpha$, where many healthy individuals exhibit extremely low levels, IL-10, sTNF-RII and IL-1Ra are readily detected in plasma of most individuals, hence can be readily measured in cohort studies. Secondly, an initial F/T cycle, virtually inevitable in practice, does not alter the results obtained upon analysis. These data are also presented in before/after plots (Additional file 1: Figure S1) to facilitate intra-individual comparisons. Finally, particularly important for studies where hundreds or thousands of samples require assessment, up to five F/T cycles had minimal impact on the levels measured.

A single exception was found among the 14 biomarkers examined. sTNF-RII differed when comparing $1 \times$ versus $5 \times \mathrm{F} / \mathrm{T}$ cycles (medians 1516 vs. $1470 \mathrm{pg} / \mathrm{mL}$, a $3 \%$ increase, $\mathrm{p}=0.002$ ). We note that comparison of never frozen plasma and $5 \times \mathrm{F} / \mathrm{T}$ was not significant (medians 1470 vs. $1516 \mathrm{pg} / \mathrm{mL}, \mathrm{p}=0.14$ ). For context, the $3 \%$ difference seen comparing one and five $\mathrm{F} / \mathrm{T}$ cycles contrasts with the $770 \%$ range $(560-4365 \mathrm{pg} / \mathrm{mL})$ in the population. Thus, while the $1 \times$ versus $5 \times \mathrm{F} / \mathrm{T}$ is statistically significant (if it had not been corrected for multiple comparisons, as would normally be performed), the biological relevance of a median $3 \%$ error in analysis elicited by freezing and thawing is minor in comparison to a 7.7 fold range in the population.

\section{Stability of systemic inflammatory biomarkers: CRP, PTX3, IL-1RACP and SST2}

We next examined a panel of widely used and emerging biomarkers of acute and chronic inflammation in humans (Fig. 3). These included CRP, historically the

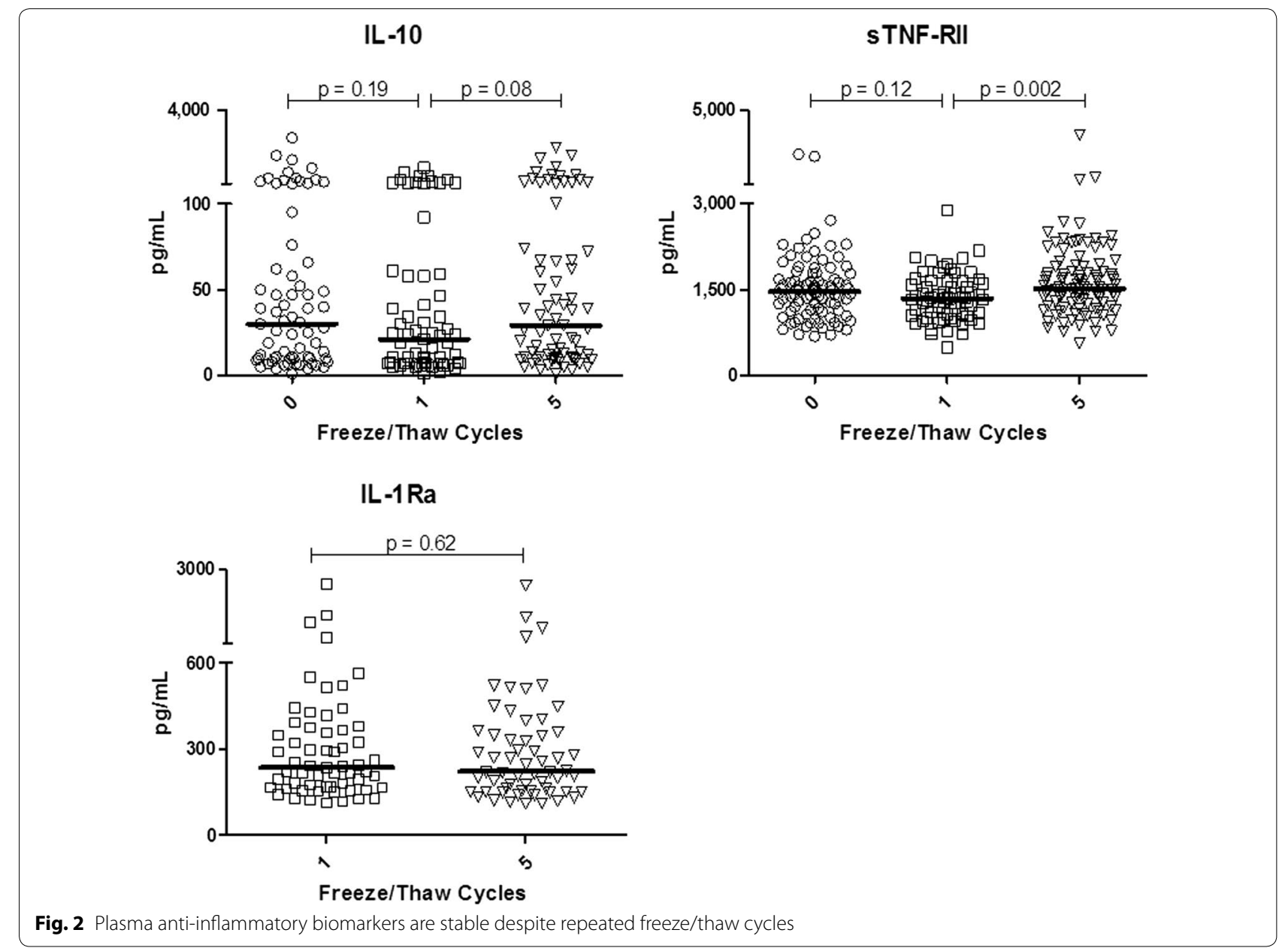


most commonly examined acute phase protein biomarker in humans; pentraxin 3 (PTX3), a systemic acute phase protein expressed in vivo more rapidly than CRP and prominent in chronic inflammation; soluble IL-1R Accessory protein (IL-1RAcP), a widely expressed protein integral to pro-inflammatory signaling and linked to asthma, autoimmunity and other inflammatory conditions; and sST2, an increasingly used predictive biomarker of inflammatory disorders and increased risk of mortality in cardiovascular disease. All were found to be ubiquitously expressed in plasma of healthy individuals. Each of these biomarkers was also stable when comparing paired data sets from freshly obtained, never frozen plasma and matched aliquots after five $\mathrm{F} / \mathrm{T}$ cycles.

\section{Serum versus plasma stability to repeated $\mathrm{F} / \mathrm{T}$ handling}

The utility of plasma versus serum in biomarker quantification has been extensively studied. Here we focus on $\mathrm{F} / \mathrm{T}$ stability of serum, examining a much broader range of immune analytes than previously reported. When serum was obtained from an individual and subjected to zero versus five repeat $\mathrm{F} / \mathrm{T}$ cycles, the values obtained were stable. This conclusion applied to pro- and antiinflammatory cytokines (Fig. 4a) as well as acute phase proteins and other biomarkers (Fig. 4b). Importantly, and as previously established, levels for a given individual in serum versus plasma are frequently independent and are not necessarily interchangeable. Thus, paired analyses of serum and plasma from the same donors frequently yielded different values for some biomarkers whereas levels of other biomarkers were indistinguishable in serum and plasma.

\section{Impact of donor age or sex on $\mathrm{F} / \mathrm{T}$ stability}

Conclusions from experimental or clinical findings are sometimes extended from one age group or sex to another in the absence of clear supporting evidence. To avoid this, we stratified the study population by age, comparing samples within young children independently from those of adults (median age 3 vs. 32). Figure 5 demonstrates that $\mathrm{F} / \mathrm{T}$ stability for the two pro-inflammatory (CCL2, IL-18; Fig. 5a) and two anti-inflammatory (IL-10, sTNFRII; Fig. 5b) molecules shown applies equally to pediatric populations. Similarly, male versus female donors did not
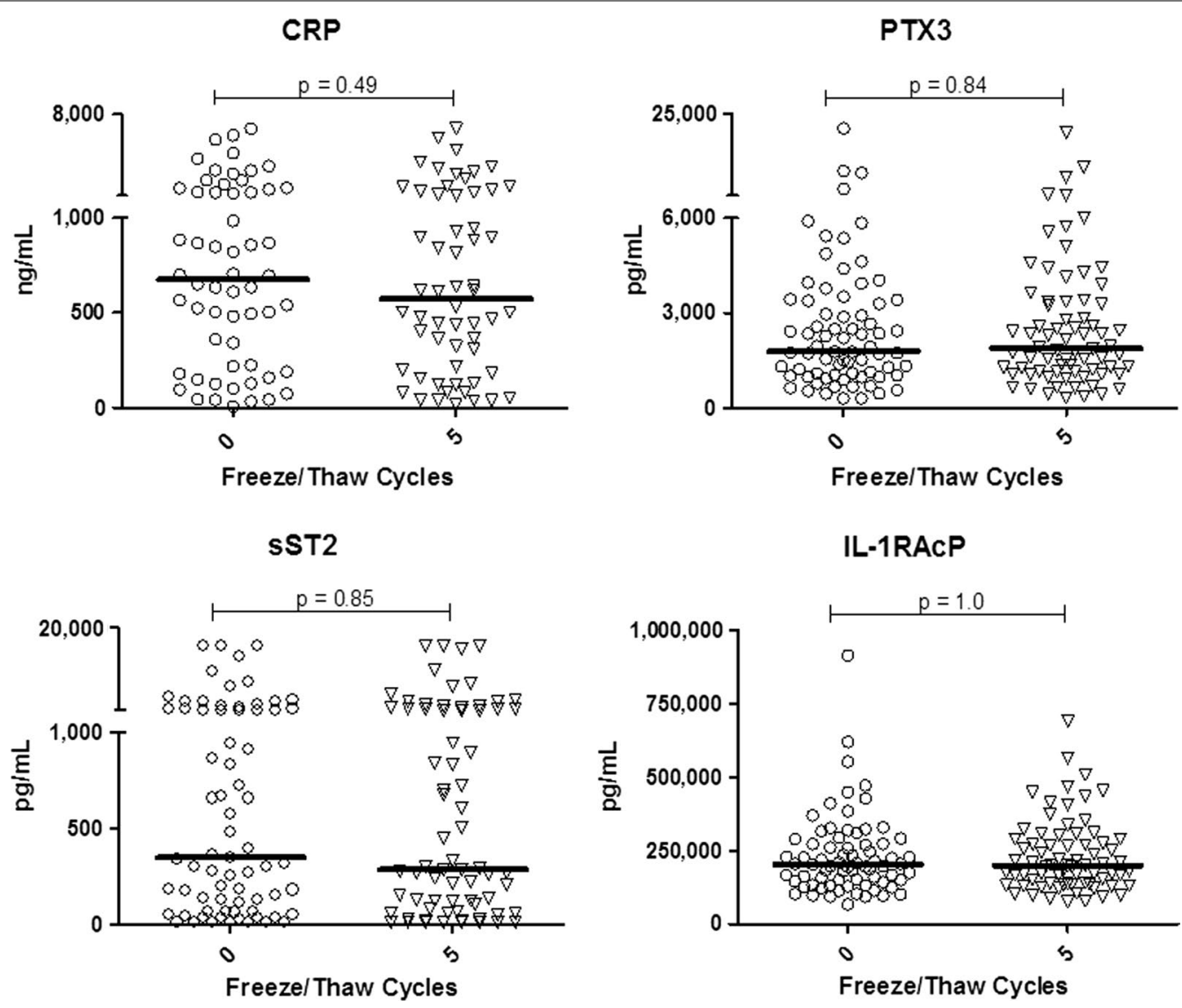

Fig. 3 Stability of acute phase proteins and related plasma inflammatory biomarkers to at least five freeze/thaw cycles 

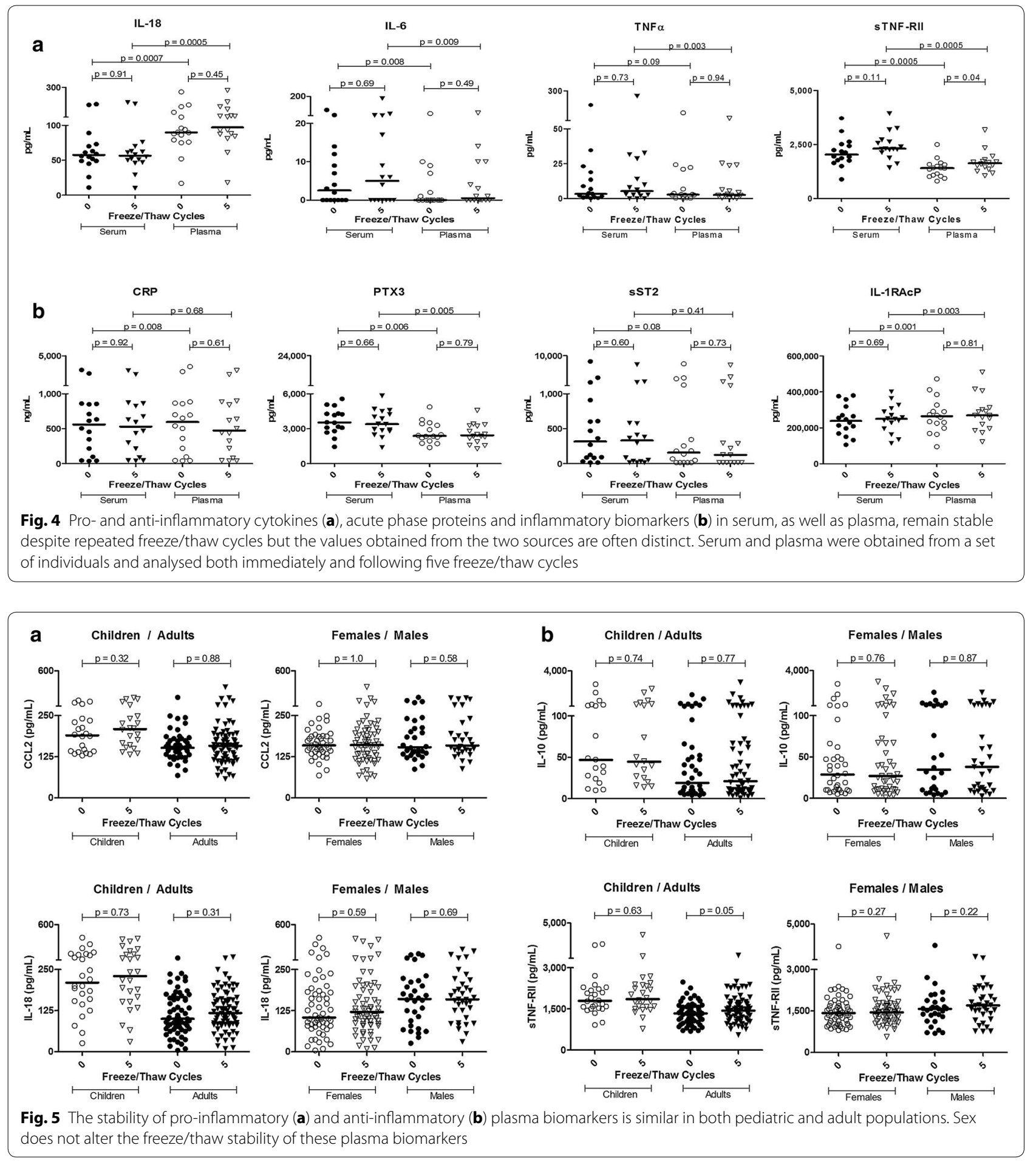

exhibit sex-attributable differences in stability of these biomarkers. CRP, PTX3, IL-1RAcP (encoded by IL1RAP) and sST2 (encoded by IL1RL1) were also stable within age and sex stratifications when comparing never frozen samples with those of five F/T cycles.
Stability of PRR-stimulated responses generated in primary culture

Acute PRR-stimulated innate immune responses provide additional insight into immune capacity. Here, primary culture supernatants were generated by stimulation 
of fresh PBMC with agonists for a representative TLR (TLR4 using LPS, $\mathrm{n}=20$ experiments, Fig. 6a) or RLR (RIG-I/MDA-5 using Lyovec conjugated poly:IC, $\mathrm{n}=30$ experiments, Fig. 6b) ligand. Both pro- and anti-inflammatory responses were stable for at least five F/T cycles.

\section{Discussion}

Human inflammatory disease research is hampered by use of a relatively small number of biomarkers to translate findings from basic biomedical research into large scale cohorts. Here we demonstrate a panel of 14 in vivo biomarkers of pro- and anti-inflammatory status that are readily quantifiable in plasma of healthy adult and pediatric populations. The results also demonstrate that no differences are evident when comparing fresh/never frozen samples with those that had undergone up to five F/T cycles. Similarly, a panel of biomarkers of innate PRRmediated activation remained stable in supernatants obtained after cell culture stimulation, directly ex vivo, despite repeated freeze thaw cycles. Thus, extending findings from individual murine and human analyses of inflammatory disorders to large human cohorts by obtaining more comprehensive innate immune signatures is readily feasible.

Prior literature on F/T stability has yielded contradictory conclusions. A representative early study (with four healthy and three HIV-infected volunteers) indicated F/T stability for the biomarkers examined [35] as did similar studies [36, 37]. Others disagreed, finding substantial sensitivity to F/T [38-41]. Using four healthy individuals, De Jager et al. [27] concluded that samples for cytokine measurements could not be subjected to repeated F/T cycles because only two of the 15 cytokines they examined did not show alterations in mean levels.

Important caveats to be aware of in interpretation of the literature include: (1) due to limited assay sensitivity, many investigators utilized samples spiked with recombinant cytokines to achieve sufficient sensitivity for the assays employed, (2) the number of individuals studied in most studies was often less than ten, (3) intraand inter-assay variation was often not provided, making it difficult to determine to what extent decreases or increases in reported cytokine levels were attributable to variability in assay or operator performance rather than F/T cycles. Moreover, in many studies, means and parametric statistical tests with significance set at 0.05 were utilized, without correction for non-parametric data distributions (log-transformation or use of non-parametric tests such as utilized above) common to small data sets or correcting for multiple comparisons (i.e. Bonferroni corrections).

When statistically significant differences are identified, it will be important to examine the scale of such differences in the context of the population being examined. For example, here, sTNF-RII levels were significantly different following one versus five $\mathrm{F} / \mathrm{T}$ cycles (medians 1516 vs. $1470 \mathrm{pg} / \mathrm{mL}$, a $3 \%$ increase, $\mathrm{p}=0.002$ ). While variance is inherent in repeated analyses of any quantitative measure, it needs to be compared in scale to the range exhibited within the study population as a whole. Thus, for sTNFRII, there is a $770 \%(560-4365 \mathrm{pg} / \mathrm{mL})$ range in values within the population studied. Similarly,

\section{a $\quad$ TLR4 stimulated}
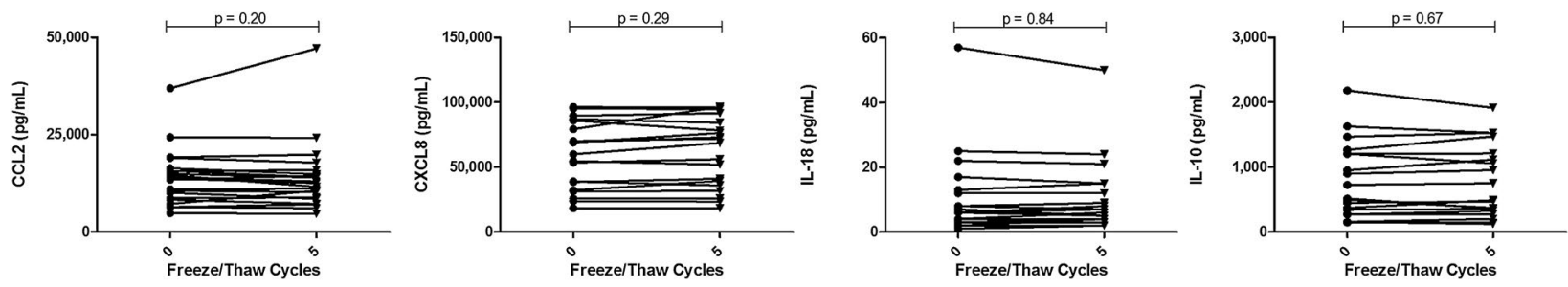

\section{b RLR stimulated}
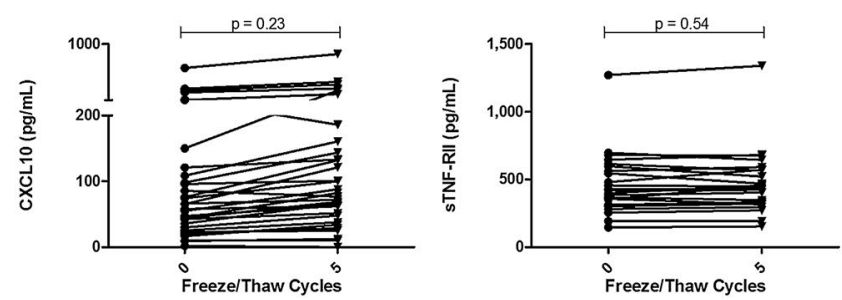

Fig. 6 Stability of pro- and anti-inflammatory biomarkers following acute PRR-mediated [TLR4 (a), RLR (b)] stimulation in vitro 
the ranges of CXCL10 ( $2500 \%$ or 25 -fold range between weakest and strongest), IL-18 (>125 fold), TNF $\alpha$ ( $>1000$ fold) and IL-10 (>3000 fold) seen in this relatively small human population $(\mathrm{n}=140)$ are important to weigh in assessing biological as well as statistical significance even when a small, statistically significant difference is contributed by sample handling.

This study has important caveats. The focus here was on the capacity to reproducibly quantify pro-/antiinflammatory biomarker concentrations ex vivo, despite virtually inevitable $\mathrm{F} / \mathrm{T}$ cycles. We did not attempt to determine biological activity (i.e. therapeutic potential), nor did we assess the stability of recombinant proteins in these assays. Other (largely controllable) factors can introduce sample variability and, depending on the study design utilized, need to be considered individually. Similarly, immune biomarkers such as Type I or III Interferons (18) that were not examined in this study may exhibit sensitivity to $\mathrm{F} / \mathrm{T}$ cycles. As additional biomarkers are added, it will be important to examine each explicitly prior to undertaking large-scale analyses. Other controllable variables, including operator error, assay variance and so forth may also introduce variance. This underlines the need for well-defined standard operating procedures (SOPs). Finally, proving that something does not occur is impossible. For that reason, this study utilized well over 100 different individuals. Variation might become detectable if 1000 or 100,000 individuals were examined, but if thousands or more samples are required to detect a difference, the size of that effect would by definition be minor.

One concern not addressed here is the long term stability of biomarkers after years of storage. Addressing this variable was beyond the scope of the present study. Use of cross-sectional study designs when comparing samples in a longitudinal cohort after three, five or seven years of storage provide an interim workaround until better data on long term stability are available. Thus, comparison can be made of samples of the same age from individuals exhibiting versus not exhibiting a given clinical phenotype is feasible, if imperfect. Certainly, prioritizing analyses to the earliest possible time point is important.

Finally, while commonly known, an important practical aspect in the implementation of such analyses should be reiterated. Logistical constraints on assay manufacturers preclude provision of constant standards from one assay to another purchased a few months or years later. This underlines the importance of establishing substantial aliquots of a single internal lab standard to be used for each assay to allow inter-assay comparison.

\section{Conclusions}

Human cohorts generate tens of thousands of biological samples, often at multiple sites. It is impossible to examine samples for all analytes simultaneously. The results above provide the foundation (and confidence) for large scale analyses of panels of inflammatory biomarkers to better understand immunological mechanisms underlying health versus disease. Specifically, the data demonstrate that an extensive panel of pro-inflammatory (CCL2, CXCL10, IL-18, TNF $\alpha$, IL-6), anti-inflammatory (IL-10, sTNF-RII, IL-1Ra), acute phase proteins (CRP, PTX3) and other biomarkers (sST2, IL-1RAcP) linked to allergy and autoimmunity and other inflammatory diseases in basic discovery research are readily detectable, even in healthy control individuals, and that they remain stable for repeat analysis despite multiple freeze thaw cycles. More frequent and more comprehensive examination of innate immune signatures would greatly enhance the value obtained from large multi-centre human cohort studies.

\section{Additional file}

Additional file 1: Figure S1. Data from representative biomarkers shown Figs. 1, 2 and 3 portrayed in a before/after format.

\section{Abbreviations \\ F/T: freeze/thaw; PBMC: peripheral blood mononuclear cells; PRR: pattern recognition receptor; RLR: RIG-like receptors; TLR: toll like receptor.}

\section{Authors' contributions}

Study concept and design: KTH, CG, ADB, ABB, RC, LL, DB; Acquisition of data: CG, RC, WPS, LL; Analysis and interpretation of data: CG, PL and KTH; Drafting of the manuscript: $\mathrm{CG}$ and $\mathrm{KTH}$; Critical revision of the manuscript for important intellectual content: all authors. All authors read and approved the final manuscript.

\section{Author details}

${ }^{1}$ Department of Immunology, University of Manitoba, Winnipeg, MB, Canada. ${ }^{2}$ Department of Pediatrics and Child Health, University of Manitoba, Winnipeg, MB, Canada. ${ }^{3}$ Children's Hospital Research Institute of Manitoba, Winnipeg, MB, Canada. ${ }^{4}$ Division of Pulmonary Medicine, Department of Medicine, University of Alberta, Edmonton, AB, Canada. ${ }^{5}$ Present Address: Community Health and Epidemiology-Saskatchewan Population Health and Evaluation Research Unit, University of Saskatchewan, Saskatoon, SK, Canada.

\section{Acknowledgements}

Not applicable.

\section{Competing interests}

The authors declare that they have no competing interests.

Availability of data and materials

All data generated or analyzed are included in this manuscript.

Ethics approval and consent to participate

University of Manitoba Health Research Ethics Board approval was obtained and written informed consent, was received from each participant or their parent prior to enrollment. 


\section{Funding}

Funding was provided by Canada Research Chairs, Canadian Institutes of Health Research and Allergen NCE. The funding bodies played no role in design of the study and collection, analysis, and interpretation of data or writing the manuscript.

Received: 24 November 2016 Accepted: 21 February 2017 Published online: 02 March 2017

\section{References}

1. Chawes BL, Stokholm J, Bonnelykke K, Brix S, Bisgaard H. Neonates with reduced neonatal lung function have systemic low-grade inflammation. J Allergy Clin Immunol. 2015;135(1450-1456):e1451.

2. Wu J, Prosperi MC, Simpson A, Hollams EM, Sly PD, Custovic A, Holt PG. Relationship between cytokine expression patterns and clinical outcomes: two population-based birth cohorts. Clin Exp Allergy. 2015;45:1801-11.

3. Pilling LC, Joehanes R, Melzer D, Harries LW, Henley W, Dupuis J, Lin $H$, Mitchell M, Hernandez D, Ying SX, et al. Gene expression markers of age-related inflammation in two human cohorts. Exp Gerontol. 2015;70:37-45.

4. Bowatte G, Lodge C, Lowe AJ, Erbas B, Perret J, Abramson MJ, Matheson $M$, Dharmage SC. The influence of childhood traffic-related air pollution exposure on asthma, allergy and sensitization: a systematic review and a meta-analysis of birth cohort studies. Allergy. 2015;70:245-56.

5. Beckhaus AA, Garcia-Marcos L, Forno E, Pacheco-Gonzalez RM, Celedon JC, Castro-Rodriguez JA. Maternal nutrition during pregnancy and risk of asthma, wheeze, and atopic diseases during childhood: a systematic review and meta-analysis. Allergy. 2015;70:1588-604.

6. Alduraywish SA, Lodge CJ, Campbell B, Allen KJ, Erbas B, Lowe AJ, Dharmage SC. The march from early life food sensitization to allergic disease: a systematic review and meta-analyses of birth cohort studies. Allergy. 2016;71:77-89.

7. Cooper PJ, Chico ME, Amorim LD, Sandoval C, Vaca M, Strina A, Campos AC, Rodrigues LC, Barreto ML, Strachan DP. Effects of maternal geohelminth infections on allergy in early childhood. J Allergy Clin Immunol. 2016;137(899-906):e892.

8. Gunaratne AW, Makrides M, Collins CT. Maternal prenatal and/or postnatal n-3 long chain polyunsaturated fatty acids (LCPUFA) supplementation for preventing allergies in early childhood. Cochrane Database Syst Rev. 2015;7:CD010085

9. Zissler UM, Esser-von Bieren J, Jakwerth CA, Chaker AM, Schmidt-Weber CB. Current and future biomarkers in allergic asthma. Allergy. 2016;71:475-94.

10. Pawankar R, Hayashi M, Yamanishi S, Igarashi T. The paradigm of cytokine networks in allergic airway inflammation. Curr Opin Allergy Clin Immunol. 2015;15:41-8

11. Song JS, You JS, Jeong SI, Yang S, Hwang IT, Im YG, Baek HS, Kim HY, Suh DI, Lee HB, Izuhara K. Serum periostin levels correlate with airway hyperresponsiveness to methacholine and mannitol in children with asthma. Allergy. 2015;70:674-81.

12. Dang TD, Tang ML, Koplin JJ, Licciardi PV, Eckert JK, Tan T, Gurrin LC, Ponsonby AL, Dharmage SC, Allen KJ. Characterization of plasma cytokines in an infant population cohort of challenge-proven food allergy. Allergy. 2013;68:1233-40.

13. Baker KF, Isaacs JD. Prospects for therapeutic tolerance in humans. Curr Opin Rheumatol. 2014;26:219-27.

14. Carmona P, Molina M, Toledano A. Blood-based biomarkers of Alzheimer's disease: diagnostic algorithms and new technologies. Curr Alzheimer Res. 2016;13:450-64.

15. Leng SX, Dandorf S, Li H, Carlson J, Hui J, Mehta SH, Piggott D, Islam S, Manwani B, Kirk GD. Associations of circulating soluble tumor necrosis factor-alpha receptors 1 and 2 with interleukin- 6 levels in an aging cohort of injection drug users with or at high risk for HIV infection. AIDS Res Hum Retrovir. 2015:31:1257-64.

16. Katial RK, Bensch GW, Busse WW, Chipps BE, Denson JL, Gerber AN, Jacobs JS, Kraft M, Martin RJ, Nair P, Wechsler ME. Changing paradigms in the treatment of severe asthma: the role of biologic therapies. J Allergy Clin Immunol Pract. 2017;5(2S):S1-S14.
17. Adamus G. Can innate and autoimmune reactivity forecast early and advance stages of age-related macular degeneration? Autoimmun Rev. 2017. doi:10.1016/j.autrev.2017.01.005.

18. Jung UJ, Seo YR, Ryu R, Choi MS. Differences in metabolic biomarkers in the blood and gene expression profiles of peripheral blood mononuclear cells among normal weight, mildly obese and moderately obese subjects. Br J Nutr. 2016;116:1022-32.

19. Bye A, Wesseltoft-Rao N, Iversen PO, Skjegstad G, Holven KB, Ulven S, Hjermstad MJ. Alterations in inflammatory biomarkers and energy intake in cancer cachexia: a prospective study in patients with inoperable pancreatic cancer. Med Oncol. 2016;33:54.

20. Scheckenbach K, Wagenmann M. Cytokine patterns and endotypes in acute and chronic rhinosinusitis. Curr Allergy Asthma Rep. 2016;16:3.

21. Tomassen P, Vandeplas G, Van Zele T, Cardell LO, Arebro J, Olze H, Forster-Ruhrmann U, Kowalski ML, Olszewska-Ziaber A, Holtappels $\mathrm{G}$, et al. Inflammatory endotypes of chronic rhinosinusitis based on cluster analysis of biomarkers. J Allergy Clin Immunol. 2016:137(1449-1456):e1444.

22. Turner PJ, Baumert JL, Beyer K, Boyle RJ, Chan CH, Clark AT, Crevel RW, DunnGalvin A, Fernandez-Rivas M, Gowland MH, et al. Can we identify patients at risk of life-threatening allergic reactions to food? Allergy. 2016:71:1241-55.

23. Pikal-Cleland KA, Rodriguez-Hornedo N, Amidon GL, Carpenter JF. Protein denaturation during freezing and thawing in phosphate buffer systems: monomeric and tetrameric beta-galactosidase. Arch Biochem Biophys. 2000;384:398-406.

24. Ceaglio N, Etcheverrigaray M, Kratje R, Oggero M. Influence of carbohydrates on the stability and structure of a hyperglycosylated human interferon alpha mutein. Biochimie. 2010;92:971-8.

25. Matheson LA, Duong TT, Rosenberg AM, Yeung RS. Assessment of sample collection and storage methods for multicenter immunologic research in children. J Immunol Methods. 2008;339:82-9.

26. Butterfield LH, Potter DM, Kirkwood JM. Multiplex serum biomarker assessments: technical and biostatistical issues. J Transl Med. 2011;9:173.

27. De Jager W, Bourcier K, Rijkers GT, Prakken BJ, Seyfert-Margolis V. Prerequisites for cytokine measurements in clinical trials with multiplex immunoassays. BMC Immunol. 2009;10:52.

28. Moffatt MF, Gut IG, Demenais F, Strachan DP, Bouzigon E, Heath S, von Mutius E, Farrall M, Lathrop M, Cookson WO. A large-scale, consortiumbased genomewide association study of asthma. N Engl J Med. 2010;363:1211-21.

29. Savenije OE, Kerkhof M, Reijmerink NE, Brunekreef B, de Jongste JC, Smit HA, Wijga AH, Postma DS, Koppelman GH. Interleukin-1 receptor-like 1 polymorphisms are associated with serum IL1RL1-a, eosinophils, and asthma in childhood. J Allergy Clin Immunol. 2011;127(750-756):e751-5.

30. Grotenboer NS, Ketelaar ME, Koppelman GH, Nawijn MC. Decoding asthma: translating genetic variation in IL33 and IL 1RL1 into disease pathophysiology. J Allergy Clin Immunol. 2013;131:856-65.

31. Savenije OE, Mahachie John JM, Granell R, Kerkhof M, Dijk FN, de Jongste JC, Smit HA, Brunekreef B, Postma DS, Van Steen K, et al. Association of IL33-IL-1 receptor-like 1 (IL1RL1) pathway polymorphisms with wheezing phenotypes and asthma in childhood. J Allergy Clin Immunol. 2011;134:170-7.

32. Subbarao $P$, Anand SS, Becker AB, Befus AD, Brauer M, Brook JR, Denburg JA, HayGlass KT, Kobor MS, Kollmann TR, et al. The Canadian Healthy Infant Longitudinal Development (CHILD) Study: examining developmental origins of allergy and asthma. Thorax. 2015;70:998-1000

33. Moraes TJ, Lefebvre DL, Chooniedass R, Becker AB, Brook JR, Denburg J, HayGlass KT, Hegele RG, Kollmann TR, Macri J, et al. The Canadian healthy infant longitudinal development birth cohort study: biological samples and biobanking. Paediatr Perinat Epidemiol. 2015;29:84-92

34. Stefura WP, Campbell JD, Douville R, Stinson MJ, Simons FE, Becker AB, HayGlass KT. Ultrasensitive ELISA for measurement of human cytokine responses in primary culture. Methods Mol Med. 2008;138:107-19.

35. Aziz N, Nishanian P, Mitsuyasu R, Detels R, Fahey JL. Variables that affect assays for plasma cytokines and soluble activation markers. Clin Diagn Lab Immunol. 1999;6:89-95.

36. Thavasu PW, Longhurst S, Joel SP, Slevin ML, Balkwill FR. Measuring cytokine levels in blood. Importance of anticoagulants, processing, and storage conditions. J Immunol Methods. 1992;153:115-24. 
37. Hosnijeh FS, Krop EJ, Portengen L, Rabkin CS, Linseisen J, Vineis P, Vermeulen R. Stability and reproducibility of simultaneously detected plasma and serum cytokine levels in asymptomatic subjects. Biomarkers. 2010;15:140-8.

38. Leemasawatdigul K, Gappa-Fahlenkamp H. Effect of storage conditions on the stability of recombinant human MCP-1/CCL2. Biologicals. 2011;39:29-32

39. Balaiya S, Grover S, Murthy RK, Chalam KV. Freezing adversely affects measurement of vascular endothelial growth factor levels in human aqueous samples. Clin Ophthalmol. 2011;5:81-5.
40. Flower L, Ahuja RH, Humphries SE, Mohamed-Ali V. Effects of sample handling on the stability of interleukin 6 , tumour necrosis factor-alpha and leptin. Cytokine. 2000;12:1712-6.

41. Guo GH, Dong J, Yuan XH, Dong ZN, Tian YP. Clinical evaluation of the levels of 12 cytokines in serum/plasma under various storage conditions using evidence biochip arrays. Mol Med Rep. 2013;7:775-80.

\section{Submit your next manuscript to BioMed Central and we will help you at every step:}

- We accept pre-submission inquiries

- Our selector tool helps you to find the most relevant journal

- We provide round the clock customer support

- Convenient online submission

- Thorough peer review

- Inclusion in PubMed and all major indexing services

- Maximum visibility for your research

Submit your manuscript at www.biomedcentral com/submit 